\title{
Effect of Oxide Thickness on GaN-based Double Gate MOSFETs
}

\author{
Safayet Ahmed and Md. Tanvir Hasan
}

\begin{abstract}
The effect of oxide thickness (EOT) on GaNbased double gate (DG) MOSFETs have been explored for low power switching device. The gate length (LG) of $8 \mathrm{~nm}$ with $4 \mathrm{~nm}$ underlap is considered. The device is turned off and on for gate voltage (VGS) of $0 \mathrm{~V}$ and $1 \mathrm{~V}$, respectively. The effective oxide thickness (EOT) is varied from $1 \mathrm{~nm}$ to $0.5 \mathrm{~nm}$ and the device performance is evaluated. For EOT $=0.5 \mathrm{~nm}$, the OFF-state current (IOFF), subthreshold slope (SS) and drain induced barrier lowering (DIBL) are obtained $2.97 \times 10-8 \mathrm{~A} / \mu \mathrm{m}, 69.67$ $\mathrm{mV} / \mathrm{dec}$ and $21.753 \mathrm{mV} / \mathrm{V}$, respectively. These results indicate that, it is possible to minimize short channel effects (SCEs) by using smaller value of EOT.
\end{abstract}

Keywords-Drain-induced barrier lowering (DIBL); Effective Oxide Thickness (EOT); GaN; OFF-state current (IOFF); Subthreshold slope (SS);

\section{INTRODUCTION}

Switching is the most important phenomenon for future device [1-3]. In order to, keep pace with the miniaturization standards, the feature size of MOSFET has been scaled down obeying Moore's law [4], which has started with $10 \mu \mathrm{m}$ halfpitch of a standard memory cell in 1971, has reached as low as $14 \mathrm{~nm}$ in 2015 [5]. The aim is to reach "more than Moore" according to the ITRS [6]. Scaling of MOSFETs has given rise to the continued improvement in both devices switching speed and density of the CMOS technology. However, they are now about to reach their physical limit [7-9]. Due to continue scaling, problems such as the short-channel effect (SCEs), parasitic capacitance, leakage current etc. arose. Double-gate MOSFETs [10] has shown superior performance in comparison to conventional single gate MOSFETs for minimizing these problems. DG MOSFETs has high transconductance and near ideal sub-threshold swing. The two gates provide good electrostatic integrity. Therefore, reduces SCEs and improve control over channel [11-14]. Silicon (Si) is the central material for the electronic devices for the last three decades. Over the years, most of the works carried out on conventional Si-based DG MOSFET [15-18]. However, as the devices getting smaller Si-based DG MOSFET has reached to its performance saturation faces many challenges

\section{Safayet Ahmed}

Lecturer; Dept. of EEE

American International University-Bangladesh (AIUB).

Dhaka, Bangladesh

e-mail: safayetahmed.s@gmail.com;
[19-21]. GaN is a probable candidate for DG MOSFETs [2223] due to its low effective mass and wide bandgap [24-25], which leads to high mobility and high saturation velocity than $\mathrm{Si}$. Therefore, it is possible to fabricate high-speed, high performance devices using GaN-based DG MOSETs.

In our previous work with GaN-based DG-MOSFET, it is reported that it's possible to attain the subthreshold slop (SS) of $62.90 \mathrm{mV} /$ decade and drain induced barrier lowering (DIBL) of $33.59 \mathrm{mV} / \mathrm{V}$ by optimizing the symmetrical underlap length with a constant gate length (LG) $12 \mathrm{~nm} \mathrm{[26].}$ The effective oxide thickness (EOT) is also another key parameter to control the OFF-state condition for future low power logic switching devices. There are some previous works that based on effect of oxide thickness [27 -28]. However, there is no report on variation of EOT for GaN-based DGMOSFETs. Therefore, more theoretical works are immense importance.

In this paper, the effect of oxide thickness on device performance has been evaluated. EOT is varied from $1 \mathrm{~nm}$ to $0.5 \mathrm{~nm}$ for device with channel length ( $\mathrm{LCH}) 12 \mathrm{~nm}$ and gate length (LG) $8 \mathrm{~nm}$ i.e. device with $4 \mathrm{~nm}$ gate underlap. The purpose of this is to find out the optimized value of EOT, that provides better device performance.

\section{Device Structure And Simulation Methodology}

The schematic structure of GaN-based DG-MOSFET is shown in Fig. 1. The channel length $(\mathrm{LCH})$ is considered 12 nm. The supply drain voltage (VDD), channel doping, and body thickness (tbody) employed throughout this paper is considered as $1 \mathrm{~V}, 1 \times 1017 \mathrm{~cm}-3$ and $5 \mathrm{~nm}$, respectively. The source to gate length (LGS) and gate to drain length (LGD) is considered as $3 \mathrm{~nm}$ in both cases. The doping of both source and drain regions is considered as $1 \times 1020 \mathrm{~cm}-3$. The Hafnium dioxide (HfO2) is employed as dielectric under the both top and bottom gates. According to the value of effective oxide thickness (EOT) the thickness of HfO2 (tin) is adjusted. At first, $4 \mathrm{~nm}$ of gate underlap is introduced by keeping the $\mathrm{LCH}$ constant at $12 \mathrm{~nm}$ and the gate length (LG) $8 \mathrm{~nm}$ for an EOT of $1 \mathrm{~nm}$. Then EOT is varied from $1 \mathrm{~nm}$ to $0.5 \mathrm{~nm}$ and results are evaluated.

The mesh structure is shown in figure 2. For all the simulation, the spacing in the $\mathrm{x}$-axis is considered $0.25 \mathrm{~nm}$ and in the $\mathrm{y}$-axis it is considered $0.1 \mathrm{~nm}$. Along each vertical slice located at the horizontal position of $\mathrm{x}$, 1-D Schrodinger 
equation is solved. All calculations are done at room temperature, $\mathrm{T}=300 \mathrm{~K}$. The non-equilibrium Green's function (NEGF) method in mode space approach has been incorporated using SILVACO ATLAS.

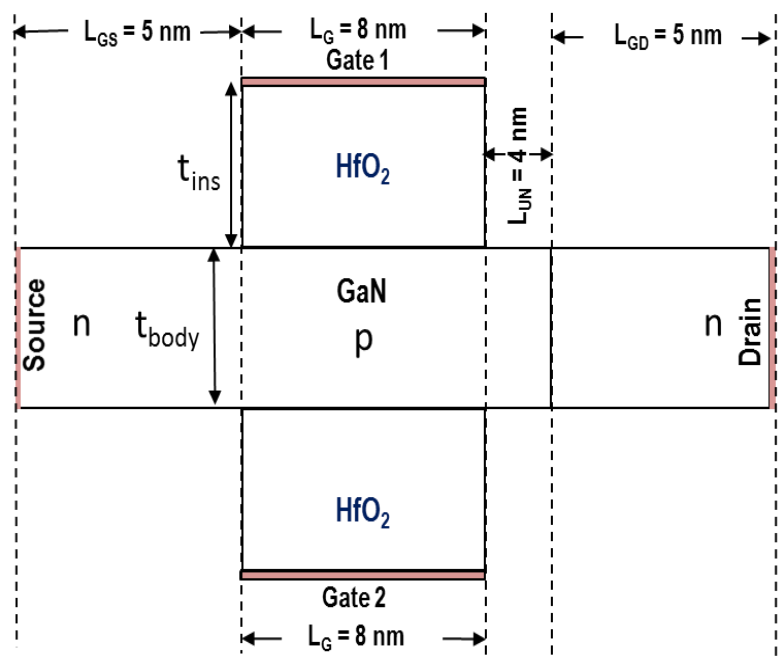

Fig. 1. Schematic structure of GaN-based DG-MOSFET.

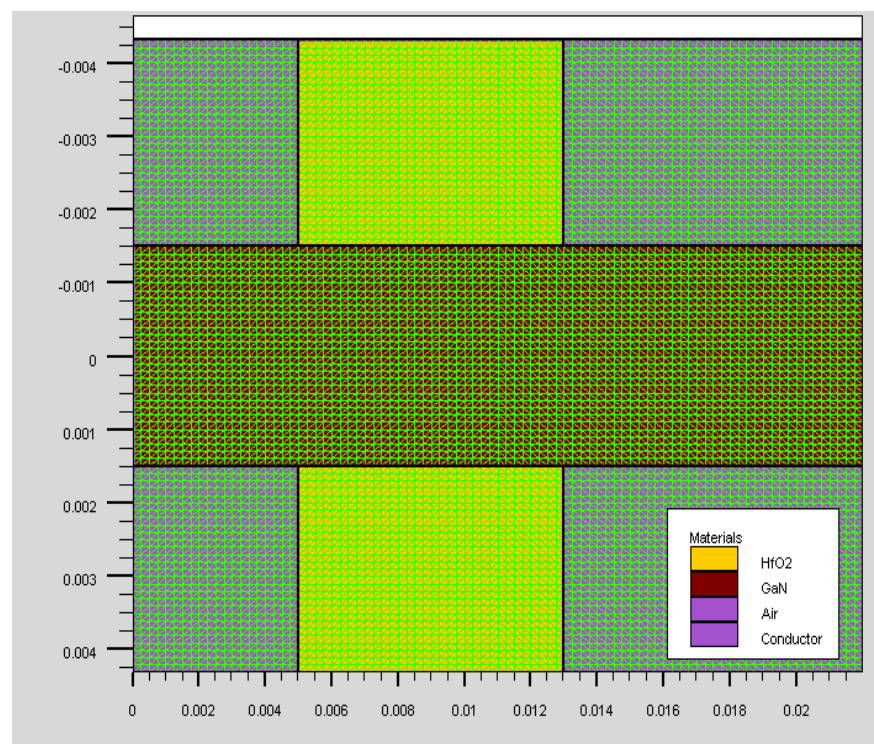

Fig. 2. Mesh Structure of GaN-based DG-MOSFET.

\section{RESULTS AND DISCUSSIONS}

In this paper, at first, the device performances have shown for a gate underlap of $4 \mathrm{~nm}$ i.e. $\mathrm{L}_{\mathrm{CH}}=12 \mathrm{~nm}$ and $\mathrm{L}_{\mathrm{G}}=8 \mathrm{~nm}$, where EOT is considered $1 \mathrm{~nm}$. Then keeping $\mathrm{L}_{\mathrm{CH}}$ and $\mathrm{L}_{\mathrm{G}}$ fixed at $12 \mathrm{~nm}$ and $8 \mathrm{~nm}$, respectively, EOT is varied from 1 $\mathrm{nm} \sim 0.5 \mathrm{~nm}$ and the device performance are analyzed.

The conduction band profile for gate underlap $4 \mathrm{~nm}$ and EOT $1 \mathrm{~nm}$ is shown in figure 3. In fig. 3 (a) the band profile is shown for OFF-state condition. In OFF-state condition the gate to source voltage (VGS) is applied $0 \mathrm{~V}$. Therefore, no change in the top of the barrier of the conduction band and it remains high. As the VGS is increased to $1 \mathrm{~V}$ for $\mathrm{ON}$-state condition the barrier height is decreased and more electron flows through the channel. Therefore, the MOSFET is in ONstate.
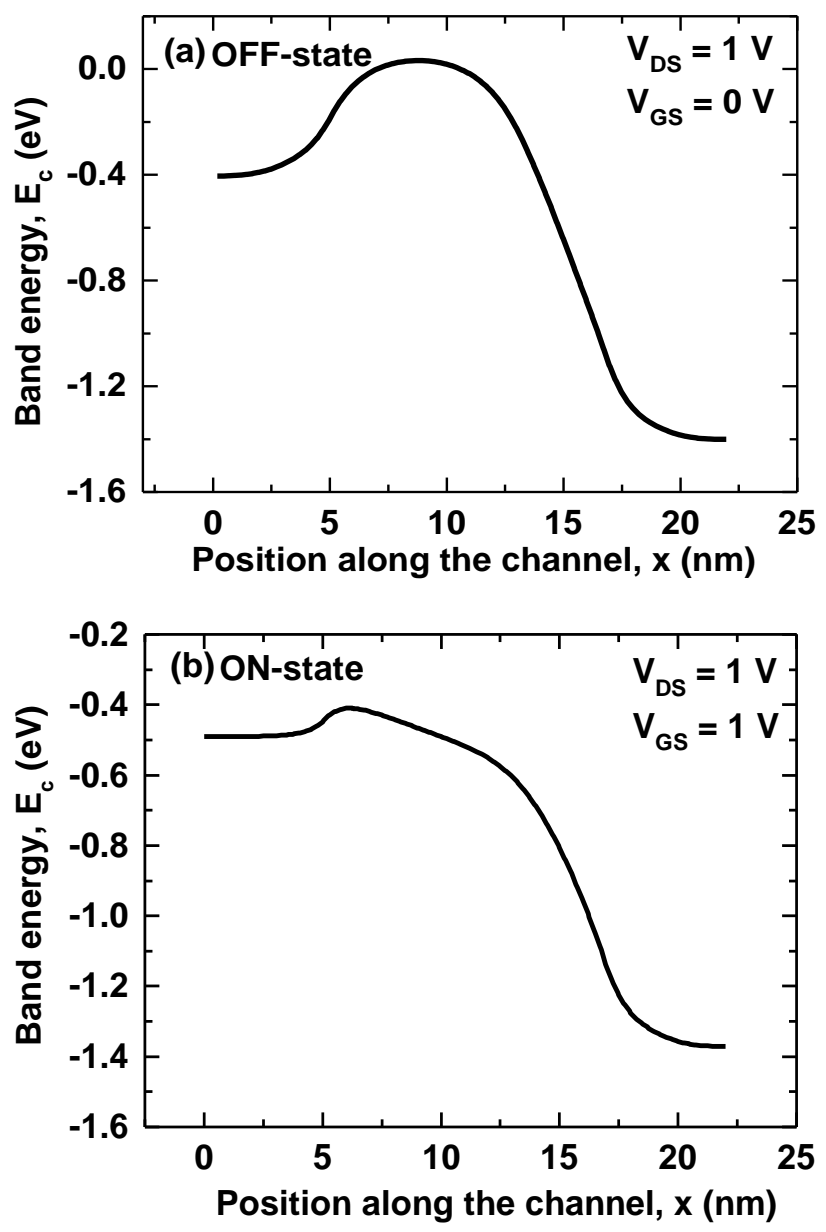

Fig. 3. Band profiles for $\mathrm{LG}=8 \mathrm{~nm}$ in the (a) OFF-state at $\mathrm{VDS}=1 \mathrm{~V}$ and $\mathrm{VGS}=0 \mathrm{~V}$ (b) ON-state at VDS $=1 \mathrm{~V}$ and VGS $=1 \mathrm{~V}$.

The transfer characteristics curves have been shown in the Fig. 4 for different drain voltage (VDS) of $1 \mathrm{~V}$ and $0.05 \mathrm{~V}$. As the drain voltage increases the $\mathrm{ON}$-state current increases.

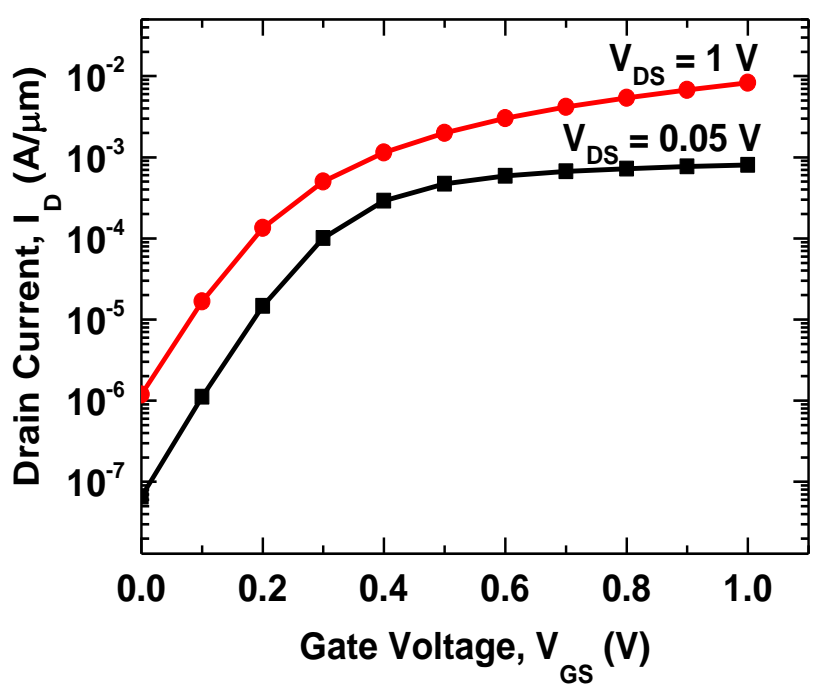

Fig. 4. Transfer characteristics curve

In Fig. 5, the dc characteristic curve is shown. With the increase of VGS, the drain current (ID) increases. The highest ID of $8.29 \mathrm{~mA} / \mu \mathrm{m}$ is obtained for $\mathrm{VGS}=1 \mathrm{~V}$ and $\mathrm{VDS}=1 \mathrm{~V}$, which is considered as ON-state current. 


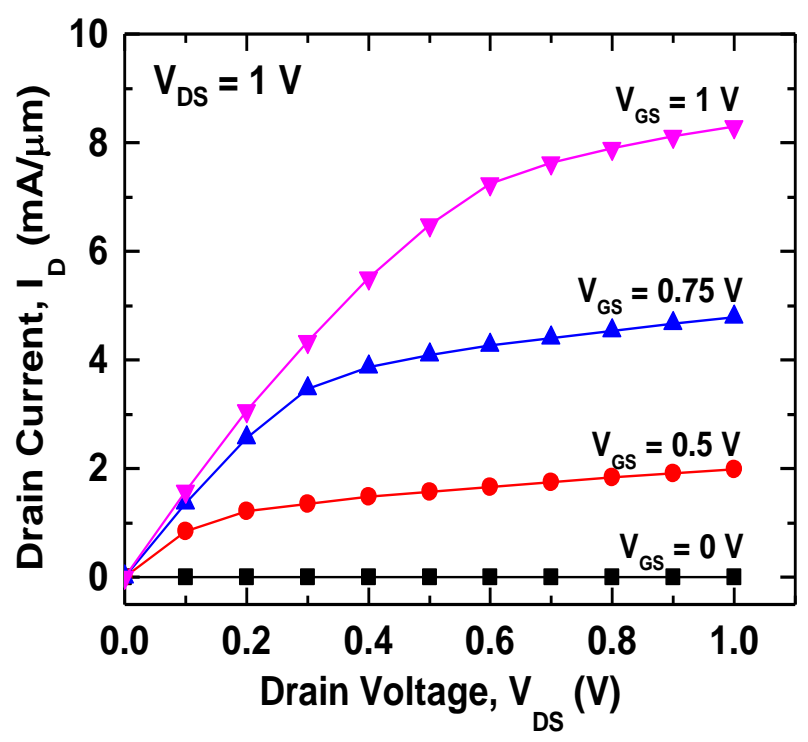

Fig. 5. Output characteristic curve for different $\mathrm{V}_{\mathrm{GS}}$.

Electric field concentration is shown in Fig. 6 (a) and (b) for OFF-state and ON-state. From the figure, it can be observed that during OFF-state condition there is no change in electric field in the channel region as no current is flowing through the channel.

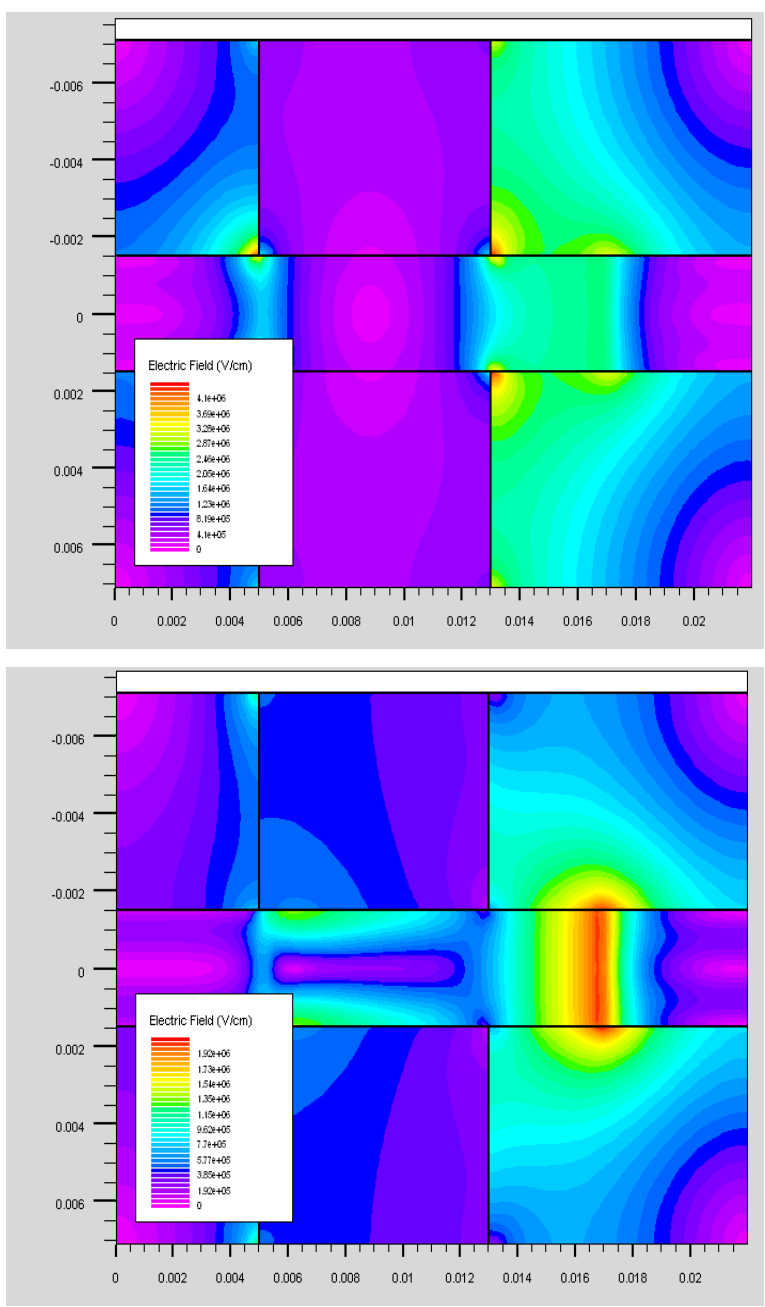

Fig. 6. Electric Field Contour (a) OFF-state condition (b) ON-state condition.
However, as the device turned on a change in electric field is observed in both top and bottom of the channel. Due to double gate two channels are created on the top and bottom of the device and due to the current flow in these channels electric field is increased in these regions.

Subthreshold slope (SS) means how much $\mathrm{mV}$ increases in gate voltage does it take to increase the output current by a factor of 10. Equation 1 describe the definition of SS. The ideal value for $\mathrm{SS}$ is $60 \mathrm{mV} /$ decade. The value of SS is found $87.27 \mathrm{mV} /$ decade for the device.

$$
S S=\frac{\Delta V_{G}}{\Delta \log I_{D}}
$$

The DIBL can be expressed as the translation of threshold voltage due to the change in drain to source voltage, as shown in equation 2. The value of DIBL is found $114.08 \mathrm{mV} / \mathrm{V}$ for device with $4 \mathrm{~nm}$ underlap.

$$
D I B L=\left[\frac{V_{T H 1}-V_{T H 2}}{V_{D S 1}-V_{D S 2}}\right]
$$

The static power consumption for the device with EOT $1 \mathrm{~nm}$ is found $1.193 \times 10^{-6} \mathrm{~W} / \mu \mathrm{m}$.

\section{A. Effect of oxide thickness}

In the previous part, it described about the result for device with gate underlap $4 \mathrm{~nm}$ and EOT $1 \mathrm{~nm}$. This part of the paper focused on the device performance based on EOT. Therefore, considering the device with gate underlap, the EOT is varied from $1 \mathrm{~nm} \sim 0.5 \mathrm{~nm}$. The relation of oxide thickness with EOT is given by equation 3 . The objective of presenting these results is to determine the optimized EOT value for the device

$$
t_{\text {ins }}=\frac{k_{\mathrm{HfO}_{2}}}{k_{\mathrm{SiO}_{2}}} \times E O T
$$

where, k_HfO2 and k_SiO2 are the di-electric constant of $\mathrm{HfO} 2$ and $\mathrm{SiO} 2$.

In Fig. 7 (a) and (b) the conduction band for OFF-state and $\mathrm{ON}$-state is shown. From the figure, it is observed that during OFF-state the barrier height is higher for smaller EOT $(0.5$ $\mathrm{nm})$. With the increase of EOT barrier height decreases. Therefore, for small EOT value, OFF-current is lower. Lower EOT means there is a less possibility of tunneling and leakage current. During ON-state, the barrier height is lower at a smaller value of EOT $(0.5 \mathrm{~nm})$. When oxide thickness is thin the gate voltage has a greater effect on the top of the barrier. Therefore, the barrier is lower for small EOT value.

The main effect of EOT on device performance is on OFFstate condition. As the value of EOT is decreased, the OFFcurrent improves. Therefore, ION $\neg / \mathrm{IOFF}$ ratio increases. $\mathrm{ION} \neg / \mathrm{IOFF}$ ratio has a significant effect on device power consumption. Hence, smaller EOT means low power consumption. In Fig. 8 the variation of ION/IOFF ratio with EOT value is shown. The highest value of ION $\neg / \mathrm{IOFF}$ ratio $(3.09 \times 105)$ is obtained for $0.5 \mathrm{~nm}$ EOT.

The dependency of SS on EOT is shown in Fig. 9. With the decrease of EOT, SCEs become prominent so that SS decreases considerably. The lowest value of SS $\quad(\sim 69.67$ $\mathrm{mV} /$ decade) is obtained for EOT $=0.5 \mathrm{~nm}$. 

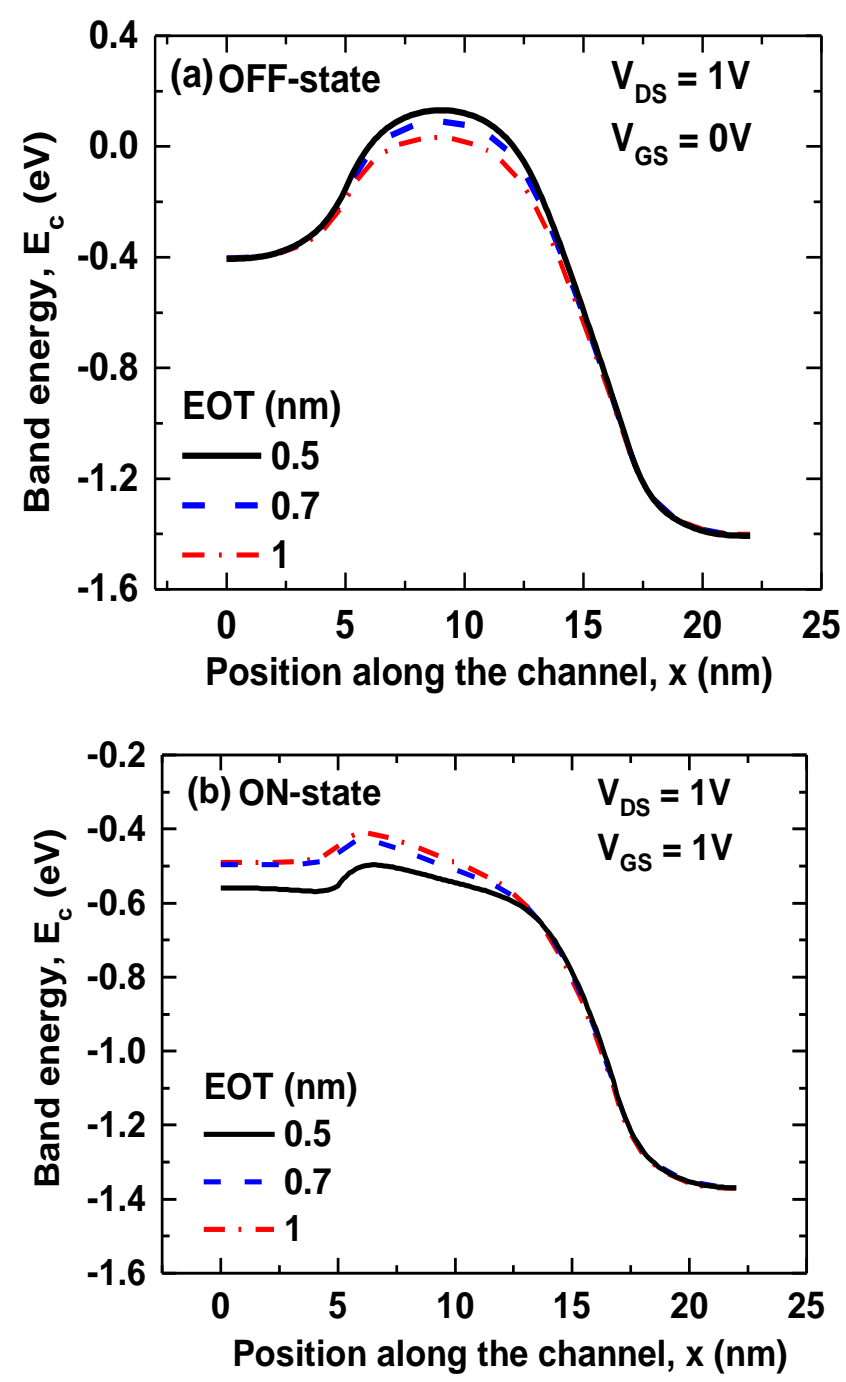

Fig. 7. Band profiles for different EOT in the (a) OFF-state at VDS $=1 \mathrm{~V}$ and $\mathrm{VGS}=0 \mathrm{~V}(\mathrm{~b})$ ON-state at VDS $=1 \mathrm{~V}$ and $\mathrm{VGS}=1 \mathrm{~V}$

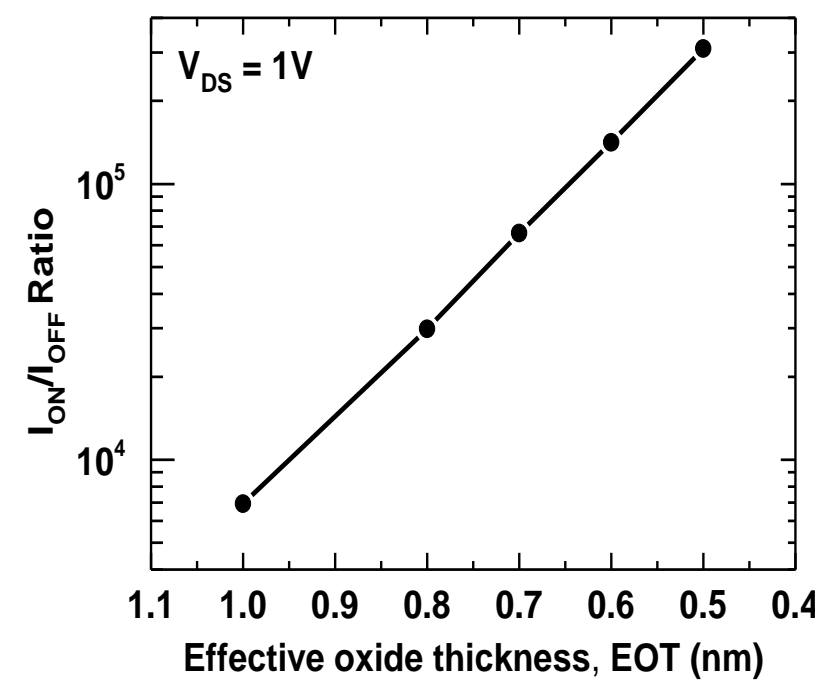

Fig. 8. Variation of ION/IOFF ratio with EOT value

In the Fig. 10, it is shown the variation of DIBL with respect to EOT. An increase in EOT increases the effect of drain potential on channel and gate losses control over the channel. Therefore, DIBL value is increased. The lowest value of DIBL $(\sim 21.753 \mathrm{mV} / \mathrm{V})$ is found for EOT $0.5 \mathrm{~nm}$.

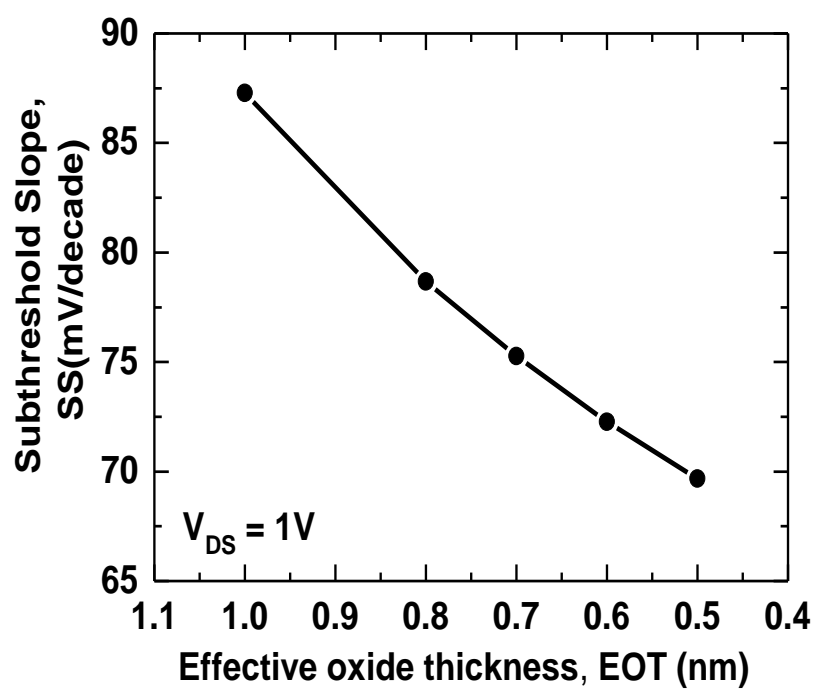

Fig. 9. Variation of SS with EOT value.

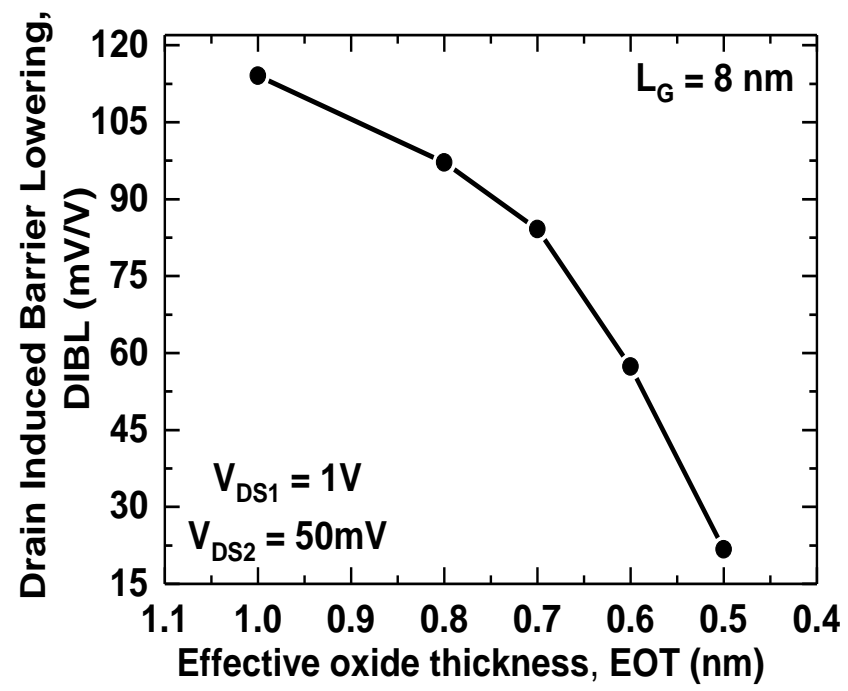

Fig. 10. Variation of DIBL with EOT value

In the Fig. 11, it is shown the variation of threshold voltage with respect to EOT. An increase in EOT decrease the threshold voltage means the OFF-state condition deteriorate. Therefore, $0.5 \mathrm{~nm}$ EOT is better for the devices.

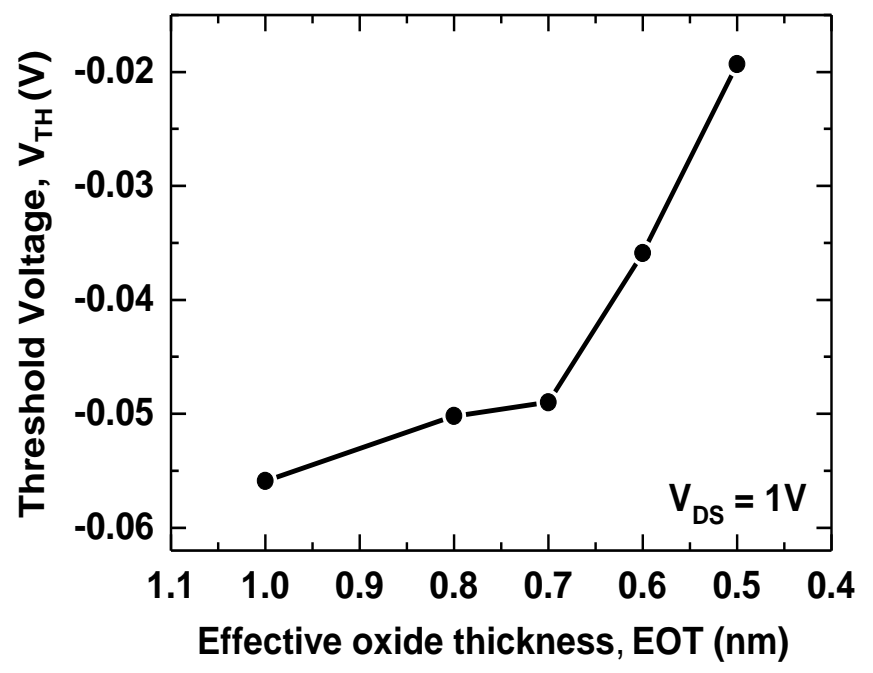

Fig. 11. Variation of threshold voltage with EOT value 
In the Fig. 12, the electric field (EF) for different EOT value has been shown. The dashed line indicates the OFF-state $(\mathrm{VGS}=0 \mathrm{~V})$ and the solid line indicates the ON-state (VGS = $1 \mathrm{~V})$. It can be determined from the figure that as the EOT value decreased from $1 \mathrm{~nm}$ to $0.5 \mathrm{~nm}$ the value of $\mathrm{EF}$ is increased. There is a higher peak on the drain side for $0.5 \mathrm{~nm}$ EOT at ON-state. The peak of EF near the drain end results in electrons entering the channel with a low initial velocity, gradually accelerating toward the drain. The maximum electron velocity is reached near the drain. Therefore, suppressing the SCEs.

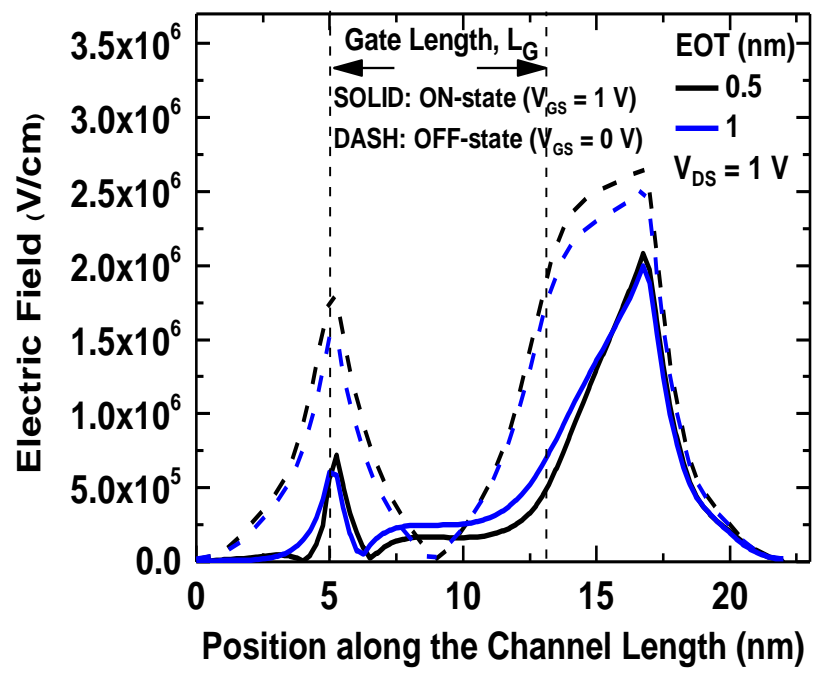

Fig. 12. Electric Field for different EOT values.

In the fig. 13, the surface potential for different EOT has been shown. As the value of EOT decreases from $1 \mathrm{~nm}$ to 0.5 $\mathrm{nm}$ the value of potential increases in the channel region. Therefore, the gate potential has more control over the channel for smaller EOT. Therefore, DIBL decreases for lower EOT and the OFF-state current decreases. Which leads to suppress in the SCEs.

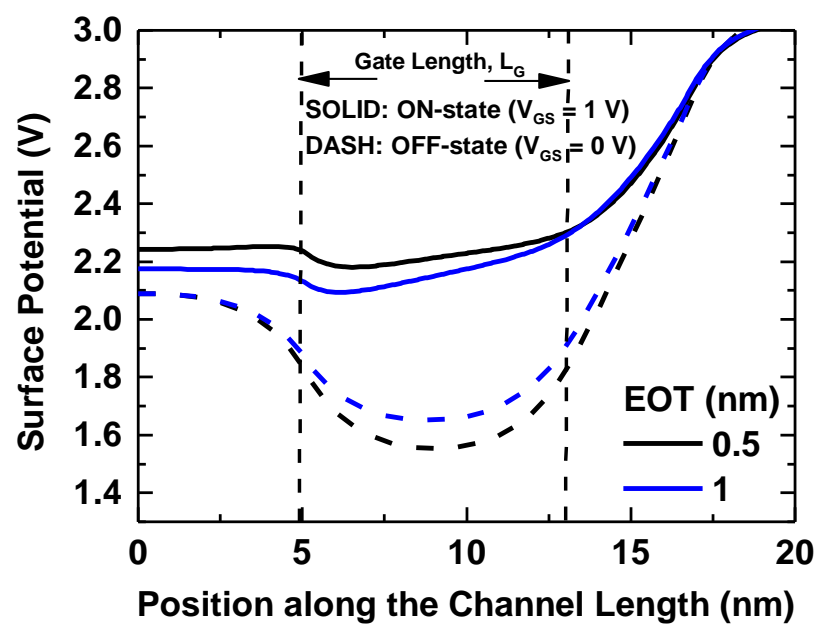

Fig. 13. Surface Potential for different EOT values.

Static Power dissipation is a significant parameter for CMOS technology. In Fig. 14 variation of static power dissipation $\left(\mathrm{I}_{\mathrm{OFF}} . \mathrm{V}_{\mathrm{DD}}\right)$ with respect to EOT has been shown. From the figure it can be obsereved that, due to the increase of $\mathrm{I}_{\mathrm{OFF}}$ with EOT the power dissipation also increases. Lowest power dissipation value $\left(\sim 2.97 \times 10^{-8} \mathrm{~W} / \mu \mathrm{m}\right)$ is obtained for
EOT $=0.5 \mathrm{~nm}$. A lower static power is highly desirable for low power and high-performance logic applications. Therefore, $0.5 \mathrm{~nm}$ EOT is appropriate to design devices that required lower power consumption.

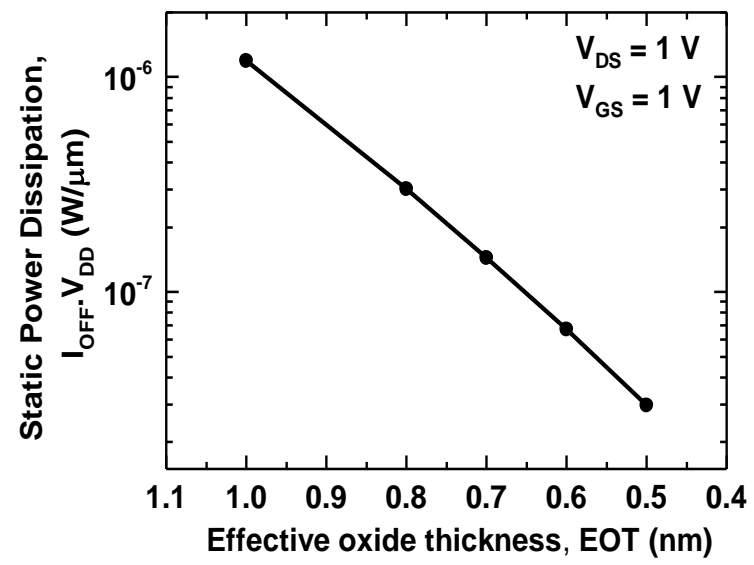

Fig. 14. Static power dissipation for different EOT values.

\section{CONCLUSIONS}

The effect of oxide thickness on GaN-based Double Gate MOSFETs has been shown with simulated results. Introducing gate underlap of $4 \mathrm{~nm}$, with fixed EOT $(1 \mathrm{~nm})$ the device performances are evaluated. Then, for the that device, EOT is varied from $1 \mathrm{~nm}$ to $0.5 \mathrm{~nm}$. The purpose is to find out the optimized value of EOT for the device. From the results, it is determined that, with the increase of EOT the SCEs become prominent. Therefore, to minimize the SCEs and improve OFF-state condition in future small devices, smaller values of EOT is desired.

\section{REFERENCES}

[1] M. Danilovic, Z. Chen, R. Wang, F. Luo, D. Boroyevich, P. Mattavelli, "Evaluation of the switching characteristics of a Gallium-Nitride transistor," in Proc. IEEE Energy Conversion Congress and Exposition 2011, pp. 2681-2688, 17-22 Sept. 2011.

[2] I. Josifovic, J. Popovic-Gerber, J.A. Ferreira, "Improving SiC JFET switching behavior under influence of circuit parasitics," in IEEE Trans. Power Electronics, vol. 27, no. 8, Aug. 2012, pp. 3843-3854.

[3] F. Merienne, J. Roudet, JL. Schanen, "Switching disturbance due to source inductance for a power MOSFET: analysis and solutions," in IEEE Power Electronics Specialists Conference 1996, vol. 2, 1996, pp. 1743-1747.

[4] G. Moore, "Cramming more components onto integrated circuits",

[5] (2013). The International Technology Roadmap for Semiconductors.[Online].Available:http://www.itrs.net/Links/2012ITRS /Home2012.htm

[6] Intel. (2017). Intel® $14 \mathrm{~nm}$ Technology. [online] Available at: http://www.intel.com/content/www/us/en/silicon-innovations/intel14nm-technology.html [Accessed 5 Jan. 2017].

[7] . Ferain, C. A. Colinge, and J.-P. Colinge, "Multigate transistors as the future of classical metal-oxide-semiconductor field-effect transistors," Nature, vol. 479, pp. 310-316, Nov. 2011.I

[8] F. Assad, Z. Ren, D. Vasileska, S. Datta, and M. Lundstrom, "On the performance limits for Si MOSFETs: A theoretical study,"IEEE Trans. Electron Devices, vol. 47, no. 1, pp. 232-240, Jan. 2000.

[9] Nikonov, D.E.; Young, I.A. "Benchmarking of Beyond-CMOS Exploratory Devices for Logic Integrated Circuits", Exploratory SolidState Computational Devices and Circuits, IEEE Journal on, Dec. 2015, Volume: 1 On page(s): $3-11$.

[10] Kunihiro Suzuki and Toshihiro Sugii, "Analytical Models for n+-p+ Double-Gate SOI MOSFET's,” IEEE Trans. Electron Devices, Vol. 42, pp. 1940-1948, 1995. 
[11] F. G. Pikus and K. K. Likharev, "Nanoscale field-effect transistors: An ultimate size analysis," Appl. Phys. Lett., Vol. 71, (25), 1997.

[12] Zhibin Ren, Ramesh Venugopal, Supriyo Datta, Mark Lundstrom, Dejan Jovanovic and Jerry G. Fossum, "The Ballistic Nanotransistor: A Simulation Study," Tech. Dig., Int. Electron Dev. Mtg., pp. 715-718, Dec. 2000

[13] Giorgio Baccarani, Susanna Reggiani, "A Compact Double-Gate MOSFET Model Comprising Quantum-Mechanical and Nonstatic Effects,” IEEE Trans. Electron Devices, Vol. 46, pp. 1656-1666, 1999.

[14] A. Rahman, M. S. Lundstrom, "A compact scattering model for the nanoscale double-gate MOSFET," IEEE Trans. Electron Devices, vol. 49, no. 3, pp. 481-489, 2002.

[15] Nikonov, D.E.; Young, I.A. "Benchmarking of Beyond-CMOS Exploratory Devices for Logic Integrated Circuits", Exploratory SolidState Computational Devices and Circuits, IEEE Journal on, Dec. 2015, Volume: 1 On page(s): 3 - 11

[16] A. Bryant, O. H. Dokumaci, X. Wang, and J. B. Johnson, "Silicon CMOS devices beyond scaling," IBM Journal of Research and Development, vol. 50, no. 4, pp. 339-361, 2006.

[17] T. Sakurai , "Perspectives of Low-Power VLSI's," IEICE Transactions on Electronics no. 4, pp. 429-436, 2004.

[18] A. Bryant, O. H. Dokumaci, X. Wang, and J. B. Johnson, "Silicon CMOS devices beyond scaling," IBM Journal of Research and Development, vol. 50, no. 4, pp. 339-361, 2006.

[19] S. Bathla and V. K. Lamba, "Comparison of Single-Gate SOI \& MultiGate SOI MOSFETs," International Journal of Emerging Technology and Advanced Engineering, vol. 3, no. 3, pp. 578-580, 2013.

[20] V. W. L. Chin, T. L. Tansley, T. Osotchan, "Electron mobilities in gallium, indium and aluminum nitrides," J. Appl. Phys. vol. 7365, no. 1994, 2016.

[21] S. Porowski, K. Pakula, C. T. Foxon, T. S. Cheng, M. Leszczynski, H. Teisseyre, T. Suski, I. Grzegory, M. Bockowski, and J. Jun, "Lattice parameters of gallium nitride Lattice parameters of gallium nitride," Appl. Phys. Lett. vol. 73, pp. 1-4, 1996.

[22] X. Jin, X. Liu, J. Lee, and J. Lee, “A continuous current model of fully depleted symmetric double-gate MOSFETs considering a wide range of body doping concentrations," Semiconductor Science and Technology, Vol. 25, no. 5, 2010.

[23] Z. Ding, G. Hu, J. Gu, R. Liu, L. Wang, and T. Tang, "An analytic model for channel potential and a subthreshold swing of the symmetric and asymmetric double-gate MOSFETs," Microelectronics J., vol. 42, no. 3, pp. 515-519, 2011.
[24] T. Paskova, D. A. Hanser, K. R. Evans, "GaN Substrates for III-Nitride Devices," Proceedings of the IEEE no. 98, pp. 1324-1338, 2010.

[25] Ki-Sik Im, Young-Woo Jo ; Jae-Hoon Lee ; Sorin Cristoloveanu ; JungHee Lee, "Heterojunction-Free GaN Nanochannel FinFETs With High Performance," in IEEE Electron Device Letters, vol. 34, no. 3, pp. 381383, 2013.

[26] Safayet Ahmed, Muhammad Shaffatul Islam and Md. Tanvir Hasan, "Effect of Symmetrical Underlap length on Device Performance of a GaN-based Double Gate MOSFET", accepeted in 5th International Conference on Informatics, Electronics \& Vision (ICIEV), 2016, Dhaka, Bangladesh.

[27] S. Das and S. Kundu, "Simulation to Study the Effect of Oxide Thickness and High- K Dielectric on Drain-Induced Barrier Lowering in N-type MOSFET," vol. 12, no. 6, pp. 945-947, 2013.

[28] S. K. Mohapatra, K. P. Pradhan, and P. K. Sahu, "Influence of High-k Gate Dielectric on Nanoscale DG-MOSFET," vol. 65, pp. 19-26, 2014.

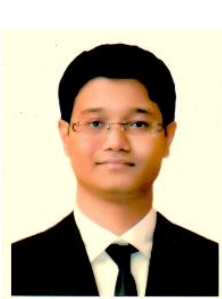

Safayet Ahmed received the Bachelor degrees in Electrical and Electronic Engineering from American International UniversityBangladesh(AIUB), in 2014. He completed his Master degree in Electrical and Electronic Engineering at American International University-Bangladesh(AIUB). He is currently working as Lecturer for the department of EEE, Faculty of Engineering at American International University-Bangladesh (AIUB).

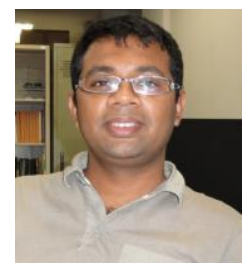

Md. Tanvir Hasan received Doctor of Engineering degree from University of Fukui, Japan in 2013. He was a Postdoctoral Research Fellow (2013-2014) in Graduate School of Engineering, University of Fukui, Japan. His research interest is focused on semiconductor devices. Currently he is working as an associate professor in the department of EEE, AIUB 